\title{
Calculation and Analysis of Transformer Transient Over-Voltage in no- Load Switching
}

\author{
Quan Fengyun ${ }^{1}$, Chen Jia1 ${ }^{1}$ Liu $\mathrm{Yu}^{2}$, Wang $\mathrm{Yu}^{2}$, He Wangling ${ }^{2}$ and Wen \\ Xishan ${ }^{2}$ \\ ${ }^{1}$ Hubei Bailianhe Pumped-storage Co., Ltd, Huanggang, China \\ ${ }^{2}$ Wuhan University, Wuhan, China
}

Key words: transformer,no-load closing,cable sheath,overvoltage,calculation

Abstract: Transformer no-load closing may result in various types of over-voltage, which may affect the stability of power system. To analyze this problem, a $500 \mathrm{kV}$ transformer no-load closing model in Bailianhe Pumped Storage Power Station is built by EMTP/ATP, the closing over-voltage on primary equipment is calculated, and the effect of closing angle on over-voltage value is analyzed. Meanwhile, the cable sheath induced voltage in transient process is analyzed. The results show that when three-phase are simultaneously closed and no resonance occurs, no-load closing over-voltage is small, and closing angle has larger impact on the overvoltage, the overvoltage multiple of transformer on low-voltage side is generally greater than that on high-voltage side. In the transformer no-load closing process, transient current of cable core is coupled to the outer sheath by inducing, which results in inducted voltage, and may cause sheath voltage in ungrounded part exceeding the insulation withstand level.

\section{Introduction}

Based on the principle of electromagnetic induction, the transformer consists cores and coils and makes the electrical energy turn into electrical energy components through the magnetic energy (Jianhua Chen, Wenhua Zhong, 2013;).Because in no-load switching process, the iron core of transformer saturates and generates operating current several times higher than the inrush current (Yilong Chen, 2011), no-load switching process is very normal but attracts much attention. However, the transformer no-load switching will generate over-voltage and its amplitude can reach several times or even more of the steady-state voltage (Zhen qiang Li, 2012).

The transformer no-load closing voltage, with little impact on the power system, is often mistaken for the internal over-voltage of the other properties by field stuff. However, with the constant improvement of the voltage level in recent years, the capacity of the power system increased. By field measure or software simulation, obvious over-voltage can be found in the noload closing process of transformer, with noise and vibration, even a partial discharge generated at the end of the high-voltage cable which is connected to the transformer (Hopkinson R H, 1965).Cables of the Bailianhe Pumped Storage Power Station have been stably running for five years, which use the XLPE high voltage dry-type of Japanese J-Power Company. Recently, when the main transformers closed in no-load condition, abnormal discharge sound and visible discharge spark have been found at the end of the cable. The causes of no-load closing over-voltage are electromagnetic oscillation over-voltage, resonance over-voltage and VFTO caused by the GIS operation. Currently, the mostly studied electromagnetic oscillations over-voltage and resonance over-voltage are considered to be the main source of the transformer no-load switching overvoltage (Jiming Lin, 2007; Mork B.A, 1994).

This paper adopts electromagnetic transient analysis software EMTP/ATP, and analyzes transient process of the transformer no-load switching over-voltage. Firstly, with the BCTRAN model and nonlinear inductance, the saturated transformer equivalent model was established, and $500 \mathrm{kV}$ transformer no-load switching circuit was built, then the study on the over-voltage generated by the transformer no-load switching transient process was carried out. Finally, the over-voltage in 
cable sheath induction during transformer no-load closing process was calculated and analyzed, and the evaluation on the influence of the voltage on the cable insulation was given.

\section{Principle of over-voltage of transformer in no-loading closing}

The transformer no-load closing operation results in high inrush current, and causes over-voltage because of oscillations or resonance. Figure 1 is a three-phase transformer connection circuit diagram. When the power source and the transformer are connected and the neutral point is grounded, the three-phase circuit can be equivalent to the single phase analysis. If the initial phase of A phase voltage $\alpha=0$, when the transformer closes, the zero state response of closing moments voltage is as follows.

$V_{T}=u_{m} \frac{n^{2}}{n^{2}-1}\left(\sin \omega \mathrm{t}-\frac{1}{n} \sin \omega_{0} \mathrm{t}\right)$

where $n=\omega_{0} / \omega ; \omega_{0}=1 / \sqrt{L_{s}\left(\mathrm{C}_{0}+C\right)} ; L_{m} \gg L_{s}$

$\omega_{0}$ is the natural oscillation frequency, $\omega$ is the system operating frequency, Ls is the power equivalent inductance, $\mathrm{C}_{0}$ is the stray capacitance of busbar, $\mathrm{R}$ is the leakage resistance, $\mathrm{Lm}$ is the excitation inductance of power transformer, $\mathrm{C}$ is the parallel values of the transformer entrance capacitance and the ground capacitance of all no-load transformer wires and electrical equipment (Zhongqing Yang, 2010).

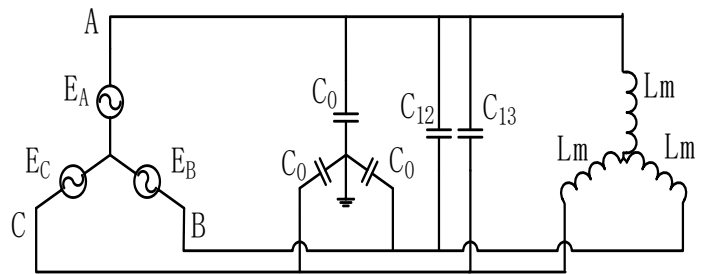

Figure 1. Three-phase transformer connection circuit diagram

In the transition process of the transformer no-load closing, the voltage contains an attenuated transient component, which is related with the transformer magnetizing inductance and entrance capacitance. Related to the closing phase angle, the closing operation over-voltage has great randomness. When the closing phase angle is near $0^{\circ}$, higher over-voltage will appear.

If the three-phase transformer closes in different periods, for triangle connection or neutral point not connected with ground in star connection of the transformer, the first closing phase and the lag closing phase produce direct electrical contact with the coupling capacitance and the magnetizing inductance, so lag closing phase has been coupled to produce oscillation over-voltage before switching in reference (Honglei $\mathrm{Xu}, 2011$ ). However, in the power grid network, the $500 \mathrm{kV}$ side winding of the transformer generally adopts the neutral point grounding in star connection, but the three-phase windings have no direct electrical connection. For three-phase core transformer, whose magnetic circuit is shown in Figure2, the three-phase can make the lag phase produce oscillation over-voltage before closing process through the magnetic circuit coupling, and may cause more serious over-voltage oscillation at the closing moment (Youqun Sun, 2004). 


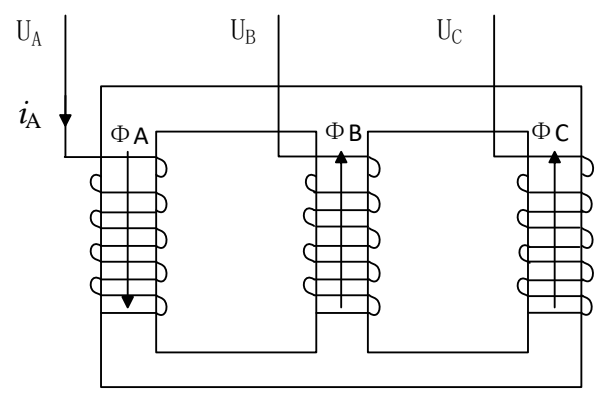

Figure 2. Transformer magnetic circuit and winding connection

If A-phase closes first, the inrush current $i_{a}$ appears. The transformer A-phase magnetic cylinders saturated and the reluctance increased. The magnetic flux generated by A-phase magnetic cylinder is $\Phi A$. When $\Phi A$ is circulated with the magnetic cylinders of $\mathrm{B}$ and $\mathrm{C}$ phase through the air gap, the voltage can induced in $\mathrm{B}$ and $\mathrm{C}$ phase system. At the closing moment, $\Phi \mathrm{A}$ imposes with sinusoidal voltage and produces more serious over-voltage. If the air gap flux is ignored and $\mathrm{B}, \mathrm{C}$ phase magnetization properties are exactly the same, $\Phi B=\Phi C=\Phi A / 2$.

The oscillation over-voltage is related to the number of the electrical equipment which is connected to the transformer and the length of the connecting wire. Due to the nonlinearity of the magnetization curve of the transformer core, the iron core is highly saturated in no-load closing process, and the inrush current which contains steady component and transient component is produced. The inrush current is biased the side of timeline and has discontinuous angle, so the frequency is rich. When wire parameters coordinate with the parameters of the transformer, which make the system self-vibration frequency similar to frequency of a particular magnetizing inrush current. It may cause the resonance over-voltage with the quite high amplitude. The no-load transformer closing circuit resistance is small, and the resonance over-voltage may continue a long period of time (Patel B, 2008; Gustavsen B, 2011).

When the no-load transformer closes, the circuit breaker fails to operate and the wire breakage causes non-full phase operation, which are more likely to cause trigger resonant over-voltage. Figure 1 is the equivalent circuit of neutral point ungrounded system. If the A phase fails to switch on, the system can be equivalent to Figure 3 , in which $\mathrm{C}_{0}{ }_{0}$ and $\mathrm{C}^{\prime \prime}{ }_{0}$ are the ground capacitance on both sides of disconnected wires.

With certain parameters and excitation conditions, the circuit can produce fundamental frequency, high frequency or frequency division resonance.

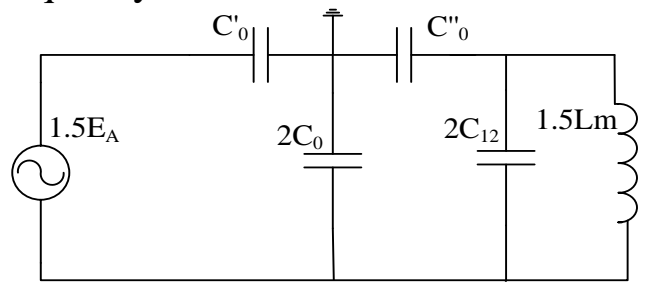

Figure 3. Equivalent circuit of open-phase system

\section{Over-voltage calculation in no-load closing of bailianhe hydropower station}

This paper, based on the electromagnetic transient analysis software ATP/EMTP, studies the overvoltage produced by $500 \mathrm{kV}$ transformer no-load closing operation situation in Bailianhe pumping energy storage power station.

\subsection{Calculate model and parameters}

The main wiring diagram is shown in Figure 4. 


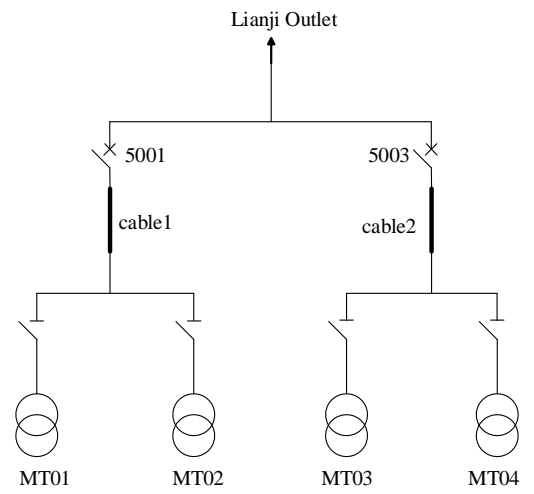

Figure 4. 500kV system wiring diagram

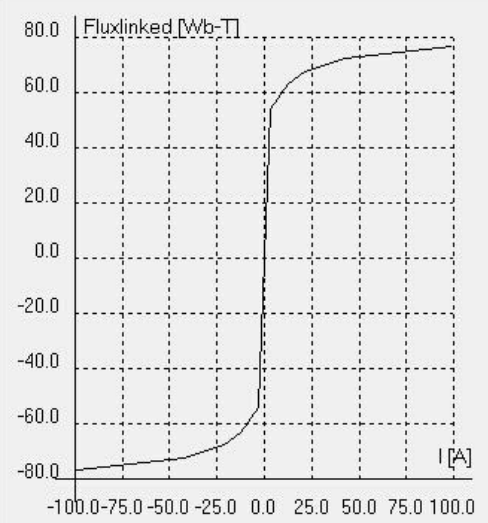

Figure 5. Transformer core magnetization curve

Main transformers No.1 and No.2 carry out switching operations through a circuit breaker 5001, so as transformers No.3 and No.4. All devices are all enclosed in the GIS except for two dry cables cable2 and cable1.

Three-phase saturation transformer uses BCTRAN model and nonlinear inductance model simulation (Jin Huang, 2010). The transformer core magnetization curve is shown in Figure 5.

\subsection{Calculation results and analysis}

The over-voltage is calculated when the No.1 main no-load transformer and the circuit breaker 5001 switch into supply power. Meanwhile, No.3 and No.4 main transformers are in normal operation and No. 2 main transformer disconnects. The circuit breaker 5001 closes when $\mathrm{t}=0$ and the A-phase closing angle is $90^{\circ}$. The high and low side voltage wave of the main transformer is shown in Figure 6.

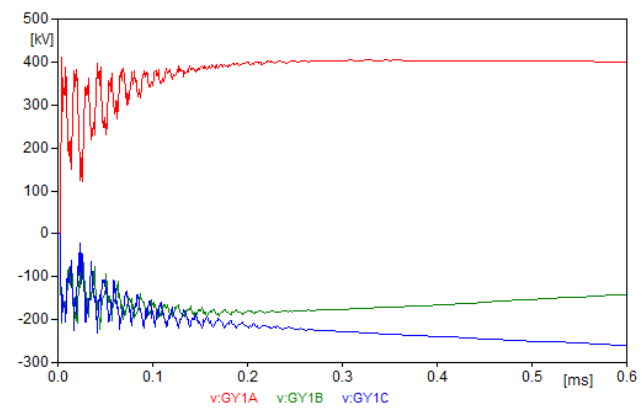

(a)Three phase waveform of main transformer high voltage side ABC 


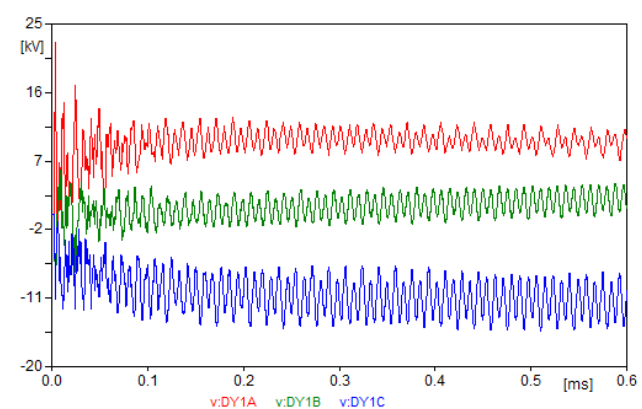

(b)Three phase waveform of main transformer low voltage side ABC

Figure 6. Waveform of main transformer no-load voltage

During the transient process, when the circuit breaker 5001 closes and sends power to the noload main transformer MT01, the no-load closing over-voltage is produced. This over-voltage transports, refracts and reflects in power station bus, cables and equipment. Most of the bus in power plant bus is mainly GIS tubular bus, which with compacted structure, covers a small area. The voltage wave refraction and reflection are more intense, causing oscillation voltage waves with high frequency in the equipment, but the voltage amplitude is small. The maximum voltage of highvoltage equipment is $450.60 \mathrm{kV}$, which is 1.1 times of the rated voltage, appearing in the outlet arrester. Merely no over-voltage occurs on high side of the main transformer, but the low side voltage is $22.51 \mathrm{kV}$, which is 1.84 times of the rated voltage. The maximum voltage on the cable core line is $422.37 \mathrm{kV}$. When the three-phase is closed at the same time and no resonance is produced, the no-load closing over-voltage is small, and merely has a significant impact on the system.

To study the effect of the closing angle on the over-voltage, the A phase closing angle was changed. The main transformer voltage is shown in Table 1.

\begin{tabular}{ccc}
\multicolumn{3}{c}{ Table 1. Effect of closing angle on Overvoltage } \\
\hline $\begin{array}{c}\text { Phase A } \\
\text { closing } \\
\text { angle }\end{array}$ & $\begin{array}{c}\text { High voltage } \\
\text { side of } \\
\text { transformer/kV }\end{array}$ & $\begin{array}{c}\text { Low voltage side } \\
\text { of } \\
\text { transformer/kV }\end{array}$ \\
\hline $90^{\circ}$ & $411.43(\mathrm{~A})$ & $22.51(\mathrm{~A})$ \\
$45^{\circ}$ & $407.90(\mathrm{C})$ & $21.63(\mathrm{C})$ \\
& $407.90(\mathrm{~A})$ & $14.33(\mathrm{~A})$ \\
$0^{\circ}$ & $407.90(\mathrm{~B})$ & $18.85(\mathrm{~B})$ \\
& $407.90(\mathrm{~A})$ & $11.86(\mathrm{~A})$ \\
\hline
\end{tabular}

*Note: $\mathrm{ABC}$ in parentheses represent three-phase

The closing angle heavily influences no-load closing over-voltage of the main transformer. When the closing angle is $90^{\circ}$, the voltage reaches the maximum, and the over-voltage of the main transformer low voltage side reaches 1.84 times of the rated voltage. The instantaneous voltage does not have transient process when the closing angle is 0 degrees, and both the main transformer high voltage side and low voltage side have no over-voltage. Hence, when main transformer noload closes, in order to reduce the effect of over-voltage on the system, effects should be made to control the closing angle at 0 degree, and to avoid closing at 90 degrees.

Also, no matter when it is closed, the over-voltage of the low voltage side of the main transformer is far greater than that of the high side. First, this is because the low side wave impedance is much larger than that of the high side. Waves spread, refract and reflect in transformer winding, and raise up voltage of low voltage side. Meanwhile, due to the rich frequency of the closing voltage, low-frequency components of the high pressure side voltage are coupled to the low pressure side through induction. High frequency components can pass through the winding stray capacity and couple to the low pressure side, resulting in larger over-voltage on low voltage side. 


\section{Cable sheath induction over-voltage calculation}

When the high voltage single core cable works, the $50 \mathrm{~Hz}$ three-phase alternating current passes through the wire core, and the transient current occurs in wire core when the transformer operates in no-load status. The changed current produces alternating magnetic field, which will produce induction voltage on the cable metal sheath. High induction voltage will directly threaten the normal work and the safety of construction personnel. "Cable Design Specification in Power Engineering" provides that the AC single-phase power cable metal sheath must be directly grounded. The normal induction voltage of any ungrounded part in the metal sheath shall comply with the following provisions: (1) When no security measures which protect people from contacting with the metal layer the voltage, the voltage shall not be greater than $50 \mathrm{~V}$. (2) In addition to the (1) item, the voltage shall not be greater than $100 \mathrm{~V}$.

The high-voltage cable sheath of Bailianhe power station is grounded in one end, and the ground resistance is $0.4 \Omega$. In steady state, the induction voltage of the cable sheath in grounding end is $6.9 \mathrm{~V}$, and the maximum induced voltage of ungrounded part is $42.2 \mathrm{~V}$, which meets the design specification. When the main transformer closes in no-load status, the waveform of cable sheath voltage in grounded end and ungrounded end are shown in figure 7.
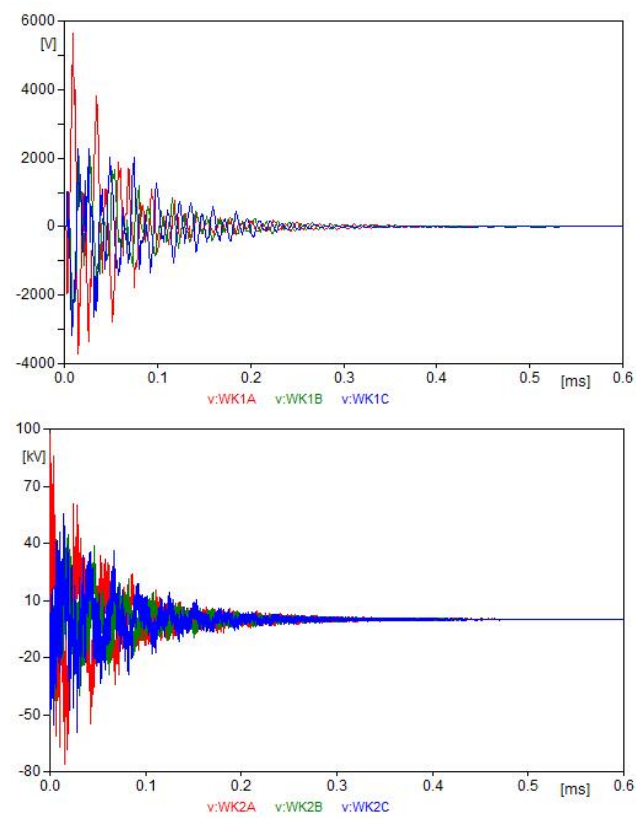

Figure 7. Induction voltage waveform of cable sheath

Due to the inductive coupling, the cable sheath voltage oscillates and its amplitude is $5.66 \mathrm{kV}$ (grounded end) and $97.6 \mathrm{kV}$ (ungrounded end). The oscillation time is $0.3 \mathrm{~ms}$.

China's relevant standards GB/T 11017, GB/Z 18890, DL/T 401, and international standard IEC 60840, IEC 62067, and other guidelines provide the insulation withstand level of the power cable sheath, which is shown in Table 2.

Table 2. Insulation withstand voltage of power cable sheath $(\mathrm{kV})$

\begin{tabular}{lllll}
\hline $\mathrm{U}_{0}$ & 110 & 220 & 330 & 500 \\
\hline $\mathrm{U}_{\text {LIW }}$ & 37.5 & 47.5 & 62.5 & 72.5 \\
\hline
\end{tabular}

Due to inductive coupling, the voltage amplitude of cable sheath ungrounded end reaches $97.6 \mathrm{kV}$, which is more than $500 \mathrm{kV}$ cable sheath insulation withstand voltage, causing sheath insulation breakdown. It is recommended that an over-voltage protector should be installed in ungrounded end to limit the sheath voltage. 


\section{Conclusions}

This paper, based on the electromagnetic transient analysis software EMTP/ATP, establishes transformer model, simulates the transformer over-voltage in no-load closing process, and analyzes the cable sheath induced voltage. The conclusions are as follows:

(1) The transformer no-load closing process may result in various types of over-voltage. If threephase simultaneously close and no resonance is produced, the over-voltage is small, which is generally less than 2 times of the rated voltage and merely has great impact on power system;

(2) The closing angle has large impact on transformer no-load switching over-voltage. When close at 90 degree, the over-voltage reaches the maximum, and the over-voltage of transformer lowvoltage side reaches 1.84 times of the rated voltage. There is no transient process in the instantaneous closing moment if closing at 0 degree, and no over-voltage appears either on the transformer high-voltage side or low-voltage side. Thus, in order to reduce this over-voltage and its impact on the system, efforts should be made to control the closing angle being 0 degree and avoid 90 degree when closing.

(3)The over-voltage of transformer low-voltage side is far greater than that of the high side. First, this is because the low side wave impedance is much larger than that of the high side. Wave spreads, refracts and reflects in transformer winding, and raises up voltage of low-voltage side. Meanwhile, due to the plentiful frequency of the closing voltage, low-frequency components of high-voltage side voltage are coupled to the low-voltage side through induction. High frequency components can couple to the low-voltage side by winding stray capacity, which result in large over-voltage on low-voltage side.

(4)When the transformer is closed in no-load status, transient current in the cable core is coupled to the outer sheath through inducing and resulting in induction voltage, which may cause the sheath voltage of ungrounded part exceeding the insulation withstand level. It is recommended that an over-voltage protector should be installed in ungrounded end to limit the sheath voltage.

\section{References}

[1] Jianhua Chen. Research on Controlled Switching of No-Load Transformers with Cable[D]. Dalian University of Technology, 2013.

[2] Wenhua Zhong. The Analysis and Reduction of Taishan Power Plant Transformer Inrush Current[D]. South China University of Technology, 2013.

[3] Yilong Chen. Analysis on the Transient Course of Traction Transformer in the Switching Proces[D]. Beijing Jiaotong University, 2011.

[4] Zhenqiang Li, Dingxie Gu,Min Dai.Analysis and Suppression Method of No-Load Ultra High Voltage Transformer Resonance Overvoltage and Inrush Current[J]. High Voltage Engineering, 2012,02:387-392.

[5] Hopkinson R H. Ferroresonance During Single-Phase Switching of 3-Phase Distribution Transformer Banks[J]. IEEE Transactions on Power Apparatus \& Systems, 1965, 84(4):289293.

[6] Jiming Lin, Xiaogang Wang, Liangeng Ban, Zutao Xiang. Resonance Overvoltage Caused by Energizing No-Load Transformers in $1000 \mathrm{kVAC}$ Transmission System[J]. Power System Technology, 2007,02:5-10.

[7] Mork, B.A, Stuehm D L. Application of nonlinear dynamics and chaos to ferroresonance in distribution systems[J]. Power Delivery IEEE Transactions on, 1994, 9(2):1009-1017.

[8] Zhongqing Yang. Investigations on Controlled Switehing of Power Transfonner[D]. Dalian University of Technology,2010.

[9] Honglei Xu, Wei Zheng, Weizhou Wang, Yong Zhi, Xueshi Li, Chao Ma, Wei Liu. Analysis of Overvoltage Factors During Primary-Side Closing of $750 \mathrm{kv}$ Transformer without Load[J].Electric Power Automation Equipment,2011,08:148-152. 
[10] Youqun Sun. Study on No-load Closing Overvoltage of Transformer in Traction Substation of Ha-da Electric Railway[D]. Tsinghua University,2004.

[11]Patel B, Das S, Roy C K, et al. Simulation of ferroresonance with hysteresis model of transformer at no-load measured in laboratory[C]// TENCON 2008 - 2008 IEEE Region 10 Conference. IEEE, 2008:1-6.

[12] Gustavsen B, Brede A P, Tande J O. Multivariate Analysis of Transformer Resonant Overvoltages in Power Stations[J]. Power Delivery IEEE Transactions on, 2011, 26(4):2563 2572.

[13]Tiecheng Lu. Hyper-Voltage of Power Systems[M].Beijing, China Waterpower Press,2009.

[14] Jin Huang. Research on Controlled Switch of No-Load Transformer[D].Xihua University,2010.

[15] GB50217-2007. Power cable design specifications in power engineering[S]. China plan press, 2007.

[16]IEC 183 - 1984. High voltage cable selection guidelines[S]. China Electric Power Press, 2002. 